\title{
Enhanced transport of relativistic electrons through nanochannels
}

\author{
Prashant Kumar Singh, Indrani Chakraborty, Gourab Chatterjee, Amitava Adak, Amit D. Lad, P. Brijesh, \\ Pushan Ayyub, and G. Ravindra Kumar* \\ Tata Institute of Fundamental Research, Mumbai 400005, India
}

(Received 25 December 2012; published 7 June 2013)

\begin{abstract}
Efficient transport of fast electrons driven by intense laser solid interaction depends crucially on optimal target design. We demonstrate a hybrid target design that incorporates two important featuresefficient generation of relativistic electrons and their unimpeded transport in dense media. The target was fabricated on a porous alumina base consisting of an array of sublambda cylindrical holes partially filled with $\mathrm{Cu}$ nanorods, such that light field propagates in the hollow channels, located ahead of the metallic fillings. The hollow array acts as an efficient source of hot electrons when driven by relativistically intense, femtosecond laser pulses and shows a 60-fold enhancement in electron flux compared to a solid target. This enhancement is ascribed to an increased penetration of laser through subwavelength pores and enhanced local electric fields. The metal doped part facilitates efficient transport of the generated electrons, due to its large background conductivity. A 4-fold enhancement in target rear side electron flux is observed compared with unfilled porous alumina.
\end{abstract}

DOI: 10.1103/PhysRevSTAB.16.063401

\section{INTRODUCTION}

The development of intense ultrashort laser pulses has opened up exciting research areas such as high-energydensity science [1], fast ignition scheme of inertial confinement fusion [2,3], compact high-energy particle accelerators [4], and ultrashort x-ray sources [5]. The interaction of intense $p$-polarized light pulses with solid targets leads to generation of relativistic fast electrons via various collisionless mechanisms [6-8]. These fast electron bunches [9] serve as carriers of laser energy from the critical surface to deep inside the target [10]. The realization of an efficient carrier depends crucially upon two aspects: (i) enhanced generation and (ii) unimpeded propagation of fast electrons through the dense plasma. The first aspect can be addressed by increasing the coupling of laser energy to the target. For polished solid targets only $30 \%-40 \%$ [6,7] of laser energy is absorbed in the plasma while the rest gets reflected. Nanostructuring the surface of the target can lead to an enhancement in the coupling efficiency by almost $100 \%$ [11] as surface plasmon excitation and lightning rod effect locally intensify the incident electric field. Similar efforts have been made to enhance the laser coupling by using other types of structured targets based on subwavelength grating [12], nanoparticles [13], nanorods [14], nanowires [15], carbon nanotubes [16], multinanolayers [17] and nanoporous materials [18]. The second aspect related to

\footnotetext{
*grk@tifr.res.in
}

Published by the American Physical Society under the terms of the Creative Commons Attribution 3.0 License. Further distribution of this work must maintain attribution to the author(s) and the published article's title, journal citation, and DOI.
PACS numbers: 52.38. $-\mathrm{r}, 52.40 \mathrm{Mj}, 52.50 . \mathrm{Jm}, 78.67 . \mathrm{Rb}$

the physics of fast electron transport depends crucially on the background electrical conductivity of the medium. The transport of relativistic mega-ampere electron currents, much higher than the Alfven limit [19] $(\sim 17 \mathrm{kA}$ for $1 \mathrm{MeV}$ electron beam), requires a nullifying return current generated from the background plasma that propagates opposite to the forward current. Net charge neutrality under such condition demands $J_{\text {return }} \simeq J_{\text {fast }}$, where $J_{\text {fast }}=$ $\left(e c n_{f}\right)$ and $J_{\text {return }}=\left(e v n_{b}\right)$ are current density of forward and return current, $n_{f}$ and $n_{b}$ are electron density of forward and return current, respectively. Since the velocity $(v)$ of the return electrons is much less than speed of light $(c)$, charge neutrality requires $n_{b} \gg n_{f}$. If the return current density is not sufficiently large, breakdown of charge neutrality will lead to creation of strong electric inhibition of forward propagating current due to electrostatic charge separation $[20,21]$. A medium with large background conductivity is therefore suitable for propagation of megaampere relativistic electron currents by enabling the smooth setting up of the return current.

So far the two above mentioned aspects have been addressed independently and an integrated scheme for optimizing both of these features in a single target is still missing. We demonstrate an innovative hybrid target design that optimizes the generation and transport of relativistic electrons. For efficient hot electron generation, the target used was a porous anodic aluminum oxide template (Whatman Anodisk), henceforth referred to as hollow PAA, containing parallel cylindrical pores of $200 \mathrm{~nm}$ diameter and $60 \mu \mathrm{m}$ length, with a density of $9 \times 10^{8}$ pores $\mathrm{cm}^{-2}$ [Fig. 1(a)]. Secondly, to improve the background conductivity for better transport, metallic copper was electrodeposited within the nanopores of the hollow PAA from a $\mathrm{CuSO}_{4}$ solution. The average length of the $\mathrm{Cu}$ nanorods 


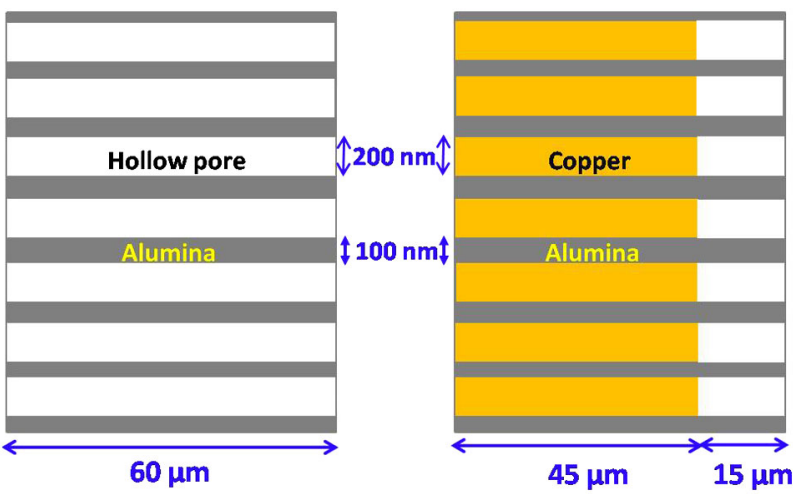

(a) Hollow PAA

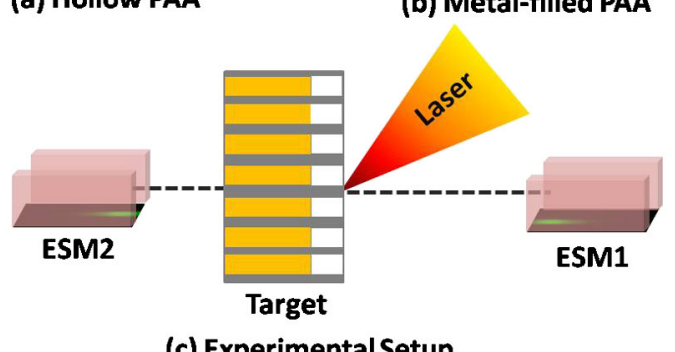

(c) Experimental Setup

FIG. 1. Schematic representation of a nanochannel target: (a) hollow PAA and (b) metal-filled PAA (hybrid target). The hybrid target is partly filled with $45 \mu \mathrm{m}$ long $\mathrm{Cu}$ nanorods. The laser incidence direction is from right to left for both targets. The sketch of experimental setup with the ESM is shown in (c).

was $\sim 45 \mu \mathrm{m}$. This hybrid target with $\mathrm{Cu}$ nanorod array sample grown inside the hollow PAA will henceforth be referred to as metal-filled PAA [Fig. 1(b)]. To determine the effect of target conductivity on the transport of fast electrons, we compare a metal-filled PAA template with a hollow PAA template on the basis of rear side electron spectra.

\section{EXPERIMENTAL SETUP}

The experiment was performed using a Ti:sapphire chirped-pulse amplification laser system, capable of delivering $600 \mathrm{~mJ}, 30 \mathrm{fs}, 800 \mathrm{~nm}$ pulses at a $10 \mathrm{~Hz}$ repetition rate. A $p$-polarized light pulse focused by a $f / 4$ off-axis parabola to a spot size of $20 \mu \mathrm{m}$ was incident on the target at $40^{\circ}$ at a peak intensity of $\sim 3 \times 10^{18} \mathrm{~W} \mathrm{~cm}^{-2}$. The insertion of an extra pockels cell after the regenerative amplifier improves the nanosecond prepulse contrast to the level of $\sim 3 \times 10^{-6}$. Two calibrated [22] electron spectrometers (ESM) were used to measure the relativistic electrons emitted from the front (ESM1) and rear (ESM2) side of the target [Fig. 1(c)]. Each has a dispersing magnet of strength $0.1 \mathrm{~T}$ and is capable of detecting electrons in the energy range $0.1-7.0 \mathrm{MeV}$. The front and rear ESM were kept at a distance of 25 and $82 \mathrm{~cm}$, respectively, along the target normal with an input aperture of $2 \mathrm{~mm}$. The dispersed electron traces on a Fuji film BAS-SR2025

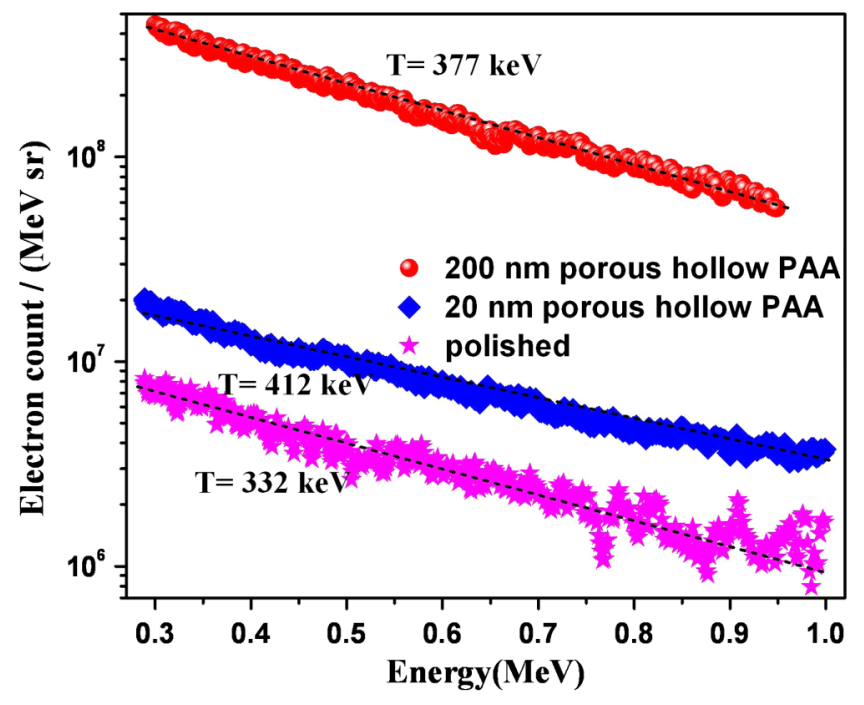

FIG. 2. Front side hot electron measurements from hollow PAA templates with 200 and $20 \mathrm{~nm}$ pore diameters, and a polished (unstructured) reference target. $\mathrm{T}$ : fast electron temperature.

image plate were read out by a Fuji phosphorimager FLA-7000.

\section{ENHANCED FAST ELECTRONS FROM NANOCHANNELS}

The hot electron spectra (averaged over 200 laser shots) at the target front for the hollow PAA and a planar glass target (used as a reference) are shown in Fig. 2. The scanning electron microscope (SEM) image of the hollow PAA target is shown in the Fig. 3. Note that the sheath potential at target-vacuum interface that modifies the electron spectra measured outside the target $[10,23]$ is similar for all targets in our comparative study and hence will not affect the inferences drawn below. The hot electron flux in the range of $0.3-1.0 \mathrm{MeV}$ is observed to be enhanced by a factor of 60 in the hollow PAA target.

This enhancement from hollow PAA can be explained in terms of (a) increased penetration of light through an array of subwavelength cylindrical cavity for a distance much greater than the skin depth and (b) enhanced local electric fields arising out of geometry $[14,24]$ and surface waves [25]. In moderate intensity laser-plasma experiments, Nishikawa et al. [18] reported an enhancement in soft $\mathrm{x}$-ray emission using a nanoporous alumina target. For a porous alumina target of pore size similar to our hollow PAA, the soft $\mathrm{x}$-ray yield increases with increasing target thickness up to $20 \mu \mathrm{m}$, after which it saturates. The enhancement of soft $\mathrm{x}$-ray bremsstrahlung arises from enhanced fast electron flux as a result of increased lasermatter interaction volume. This indicates that the laser energy penetrates as deep as $20 \mu \mathrm{m}$, much greater than the skin depth for $800 \mathrm{~nm}$ light. As the light field penetrates 

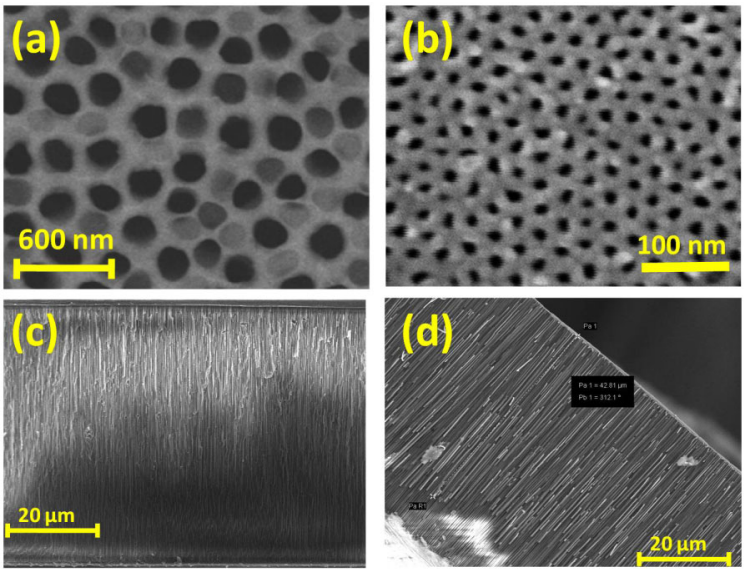

FIG. 3. SEM images of surface of hollow PAA templates of diameter (a) $200 \mathrm{~nm}$ and (b) $20 \mathrm{~nm}$, respectively. Cross section SEM image of hollow PAA (c) and metal-filled PAA (d) targets.

several times the skin depth into the nanopores, the interaction volume between laser and the target is greatly enhanced. This leads to efficient coupling of light into the matter and produces a flux much higher than in a plane target. The enhancement of fast electron flux, based on increased light penetration, can be estimated for our experiments. The ratio of laser-matter interaction volume $(R)$ for hollow PAA and planar targets (considering the same laser focal spot) is given as $R=\delta_{\text {PAA }} / \delta_{\text {planar }}$, where $\delta_{\text {PAA }}$ and $\delta_{\text {planar }}$ are the light penetration depth for hollow PAA and planar targets, respectively. For $800 \mathrm{~nm}$ light pulse, $\delta_{\text {planar }}$ given as $c / \omega_{p}$ is roughly $130 \mathrm{~nm}$, whereas $\delta_{\mathrm{PAA}}$ is about $20 \mu \mathrm{m}$ [18]. Hence we obtain $R \sim 150$. However, considering that only $30 \%$ of the target surface consists of nanopores, the effective enhancement factor of laserinteraction volume is $R_{\text {eff }} \sim 45$. Note that this value agrees quite well with our observed enhancement in the hot electron flux from the hollow PAA target as compared to a plane target (Fig. 2).

\section{SIMULATION OF LIGHT PROPAGATION IN NANOCHANNELS}

For a simple illustration of the electric field enhancement, we take recourse to an open-source finite-difference time domain software package (MEEP) to simulate the interaction of light with nanochannels. The nanochannel structure used in the simulation consists of $4 \mu \mathrm{m}$ long arrays of $100 \mathrm{~nm}$ thick metallic wires separated by $200 \mathrm{~nm}$, similar to the hollow PAA templates [Fig. 4(a)]. By launching a monochromatic $(800 \mathrm{~nm})$ point source normal to the nanochannels, a strong localization of the electric field is distinctly observed in the vacuum gaps between metal wires (Fig. 4). Unlike the very small skin depth $(\sim 100 \mathrm{~nm})$ of visible light in a metal slab, the simulation shows a strong transmission of light at the

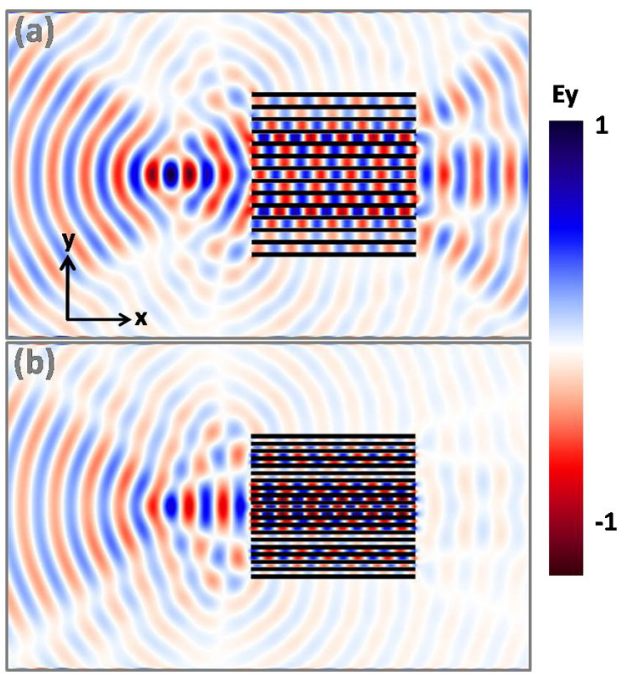

FIG. 4. Finite-difference time domain (MEEP) simulation for light transmission through nanochannels of (a) $200 \mathrm{~nm}$ and (b) $80 \mathrm{~nm}$ hollow channels. The length and width of the targets in (a) and (b) were $4 \mu \mathrm{m} \times 4 \mu \mathrm{m}$ and $4 \mu \mathrm{m} \times 3.5 \mu \mathrm{m}$, respectively.

rear of $4 \mu \mathrm{m}$ metallic nanochannels. The enhanced localization of the electric field coupled with increased light interaction region leads to enhanced generation of the electron flux observed for hollow PAA in Fig. 2. The light flux transmitted through the nanochannels was found to increase by increasing the separation between the nanowalls (Fig. 4). For example, the light flux energy is enhanced by a factor of 14 after increasing the pore separation from $80 \mathrm{~nm}$ to $200 \mathrm{~nm}$. Consistent with this picture, we observed a 17 -fold enhancement in the integrated flux of the relativistic electrons, on increasing the pore size by an order of magnitude (from $20 \mathrm{~nm}$ to $200 \mathrm{~nm}$ ), as shown in Fig. 2. It may be argued that the observed enhancement of fast electrons in hollow nanochannels will not be applicable if the prepulse driven expanding plasma is sufficiently large enough to fill the hollow channels. However, the measured enhancement values in fast electron flux for both 200 and $20 \mathrm{~nm}$ hollow templates indicate that preplasma is not significant at the intensities used in the experiment.

\section{FAST ELECTRON TRANSPORT IN METAL- FILLED NANOCHANNELS}

After demonstrating the nanochannel PAA template as an efficient source for hot electron enhancement, we have studied the transport of fast electrons in media of similar geometry but of different conductivity, namely, hollow and metal-filled PAA templates. To specifically infer the influence of background medium on the transport, we must have the same source of fast electrons being launched into the target. This was ensured by keeping the geometry of the laser-matter interaction region the same in both 


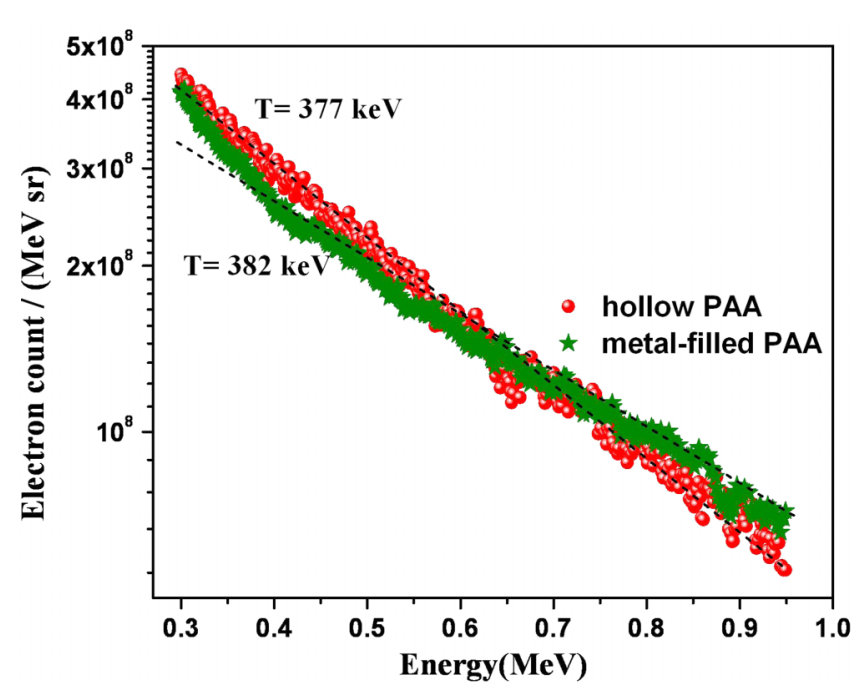

FIG. 5. Front side hot electron measurement by ESM1 for hollow PAA and metal-filled PAA target.

cases. As the laser penetration depth in the porous alumina target is nearly $20 \mu \mathrm{m}$, the initial $15 \mu \mathrm{m}$ length of the metal-filled PAA target was devoid of any $\mathrm{Cu}$ filling, as shown in the Fig. 1. The hot electron spectra measured at the front of both targets (hollow and metal-filled PAA) are shown in the Fig. 5. The hot electron temperature and integrated flux are quite similar for both cases, validating that the fast electron source is similar in both targets. A comparison of the subsequent transport of the source electrons through these targets was done by measuring electron spectra at the rear of the targets. The spectra measured by ESM2 for hollow and metal-filled PAA are shown in Fig. 6. The observed hot electron temperatures for two targets were $232 \mathrm{keV}$ and $325 \mathrm{keV}$, respectively. Thus, the metalfilled nanochannel target produced nearly 1.5 times hotter electron temperature and 4 times higher electron flux in the

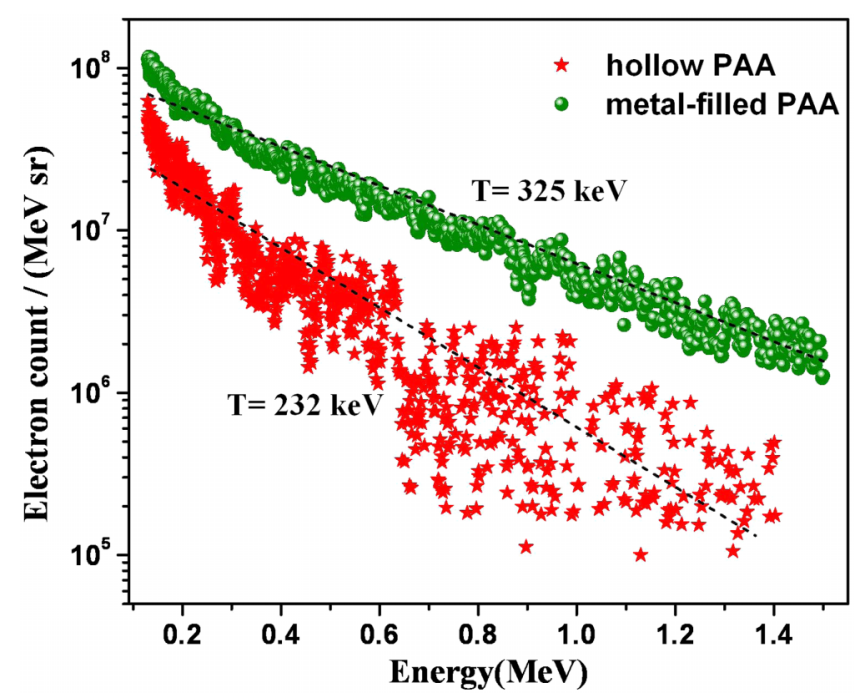

FIG. 6. Rear side hot electron measurement for hollow and metal-filled PAA targets.
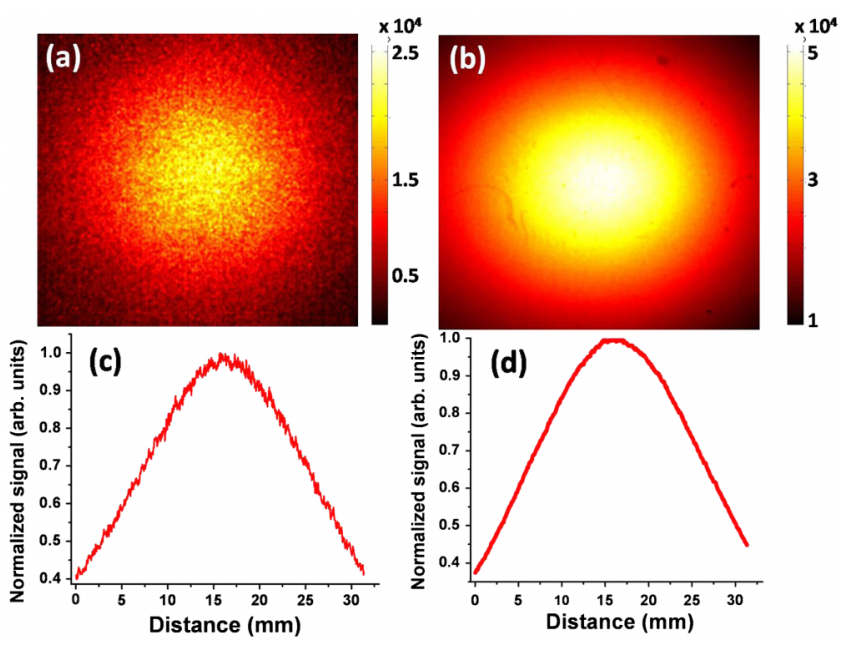

FIG. 7. The spatial distribution of fast electrons $(>70 \mathrm{keV})$ measured at the rear of the (a) hollow PAA and (b) metal-filled PAA targets. The line cut of the image (a) and (b) are shown in (c) and (d), respectively.

energy window of $(0.2-1.4) \mathrm{MeV}$ than the hollow PAA template.

The observed enhanced rear side hot electron flux can be attributed to either unimpeded transport of the fast electrons inside the target and/or collimation of electron beam as they are propagating in the vacuum. A hot electron divergence measurement was performed at the target rear to see the level of collimation of hot electrons in vacuum for both hollow and metal-filled PAA targets. An image plate (IP, BAS-2025), having a high dynamic range for electron flux, was placed at a distance of $24 \mathrm{~mm}$ from the interaction point, capturing electrons escaping from the target rear at laser pulse of intensity $3 \times 10^{18} \mathrm{~W} \mathrm{~cm}^{-2}$. To prevent optical noise, low energy electrons, and $\mathrm{x}$ rays from flooding the signal, the image plate was wrapped with $45 \mu \mathrm{m}$ thick aluminum foil, which stops electrons up to $70 \mathrm{keV}$, protons up to $2 \mathrm{MeV}$, and $\mathrm{x}$ rays up to $5 \mathrm{keV}$. The hot electron (energy $>70 \mathrm{keV}$ ) angular divergence (FWHM) was measured to be $36^{\circ}$ for both hollow and metal-filled PAA targets as shown in Fig. 7. Since the angular divergence is the same for both targets, the role of beam divergence in the measured hot electron flux enhancement for the metal-filled PAA target (Fig. 6) can be ruled out. Therefore the enhancement can only be attributed to smoother, unimpeded transport of fast electrons in a metal-filled PAA target, compared to a hollow PAA target as corroborated by the 3 -fold enhanced hot electron flux observed in the image plate data (Fig. 7) for metal-filled PAA. This is also evident in the scattered nature of the electron flux data, mainly due to insufficient signal-to-background ratio, from hollow PAA as opposed to a smoother profile for metal-filled PAA (Figs. 6 and 7). 


\section{DISCUSSION}

Fast electrons streaming through the dense media can suffer losses due to collisions and electrical inhibition. For the propagation of relativistic mega-ampere currents inside a media, the electrical inhibition is much stronger than collisional loss [20]. This can be shown by a simple calculation based on our experimental parameters. The energy loss of hot electrons due to collisions is proportional to the areal mass density of the target, $\rho \times l$, where $\rho$ is the mass density of the target and $l$ is the thickness of the target. Since the fast electrons traverse the same geometrical distance in both hollow and metal-filled PAA templates, the ratio of collisional loss depends only upon their mass density. As the mass density ratio of hollow and filled alumina, $\left(\rho_{\text {hollow-PAA }}\right) /\left(\rho_{\text {filled-PAA }}\right) \simeq 0.8$, the electron flux at the rear of hollow PAA should be 1.3 times larger than metal-filled PAA. However, our data show that fast electron flux at the rear of metal-filled PAA target is more than 4 times that of hollow dielectric PAA, which confirms that the electrical inhibition is playing a dominant role. The penetration depth $(L)$ of fast electrons [26] inside media due to electrical inhibition alone is given as $L=3 \times 10^{-3} \sigma\left(T_{h}\right)^{2}\left(I_{\mathrm{abs}}\right)^{-1} \mu \mathrm{m}$, where $\sigma$ is the conductivity in units of $10^{6}(\Omega \mathrm{m})^{-1}, T_{h}$ is the temperature of fast electrons in $\mathrm{keV}$, and $I_{\mathrm{abs}}$ is the absorbed laser intensity into the target in units of $10^{17} \mathrm{~W} / \mathrm{cm}^{2}$. For an identical source of hot electrons launched into the hollow and metal-filled PAA (Fig. 5), the penetration depth $L$ will only depend upon the conductivity of the media. For metal-filled PAA, $\mathrm{Cu}$ has an excellent conductivity of $\sim 10^{6}(\Omega \mathrm{cm})^{-1}$ at room temperature. The conductivity of copper plasma is similarly much larger than that of dielectric plasma due to incomplete ionization in the latter target. The setting up of a return current is therefore quite feasible in the $\mathrm{Cu}$ target, and leads to an appreciable neutralizing beam, required to nullify the charge separation. In the case of hollow dielectric PAA, having practically zero conductivity $\left[\sim 10^{-14}(\Omega \mathrm{cm})^{-1}\right]$ at room temperature, the neutralizing return current is generated mostly via field ionization created by a self-consistent electric field of fast electrons. This ionization leads to dissipation of beam energy of fast electrons and reduction in flux [27]. As shown in Fig. 6, the hot electrons suffer a drastic reduction both in temperature and flux while traveling in the hollow PAA. The setting up of the return current via field ionization might lead to an increase in the conductivity of the dielectric media. However, a study [28] of the background conductivity, based on a diffusion time scale of a measured magnetic field, gives an order of magnitude higher conductivity for a metal target compared to a dielectric, for an identical source of fast electrons. An order of magnitude smaller conductivity of the dielectric media will lead to an order of magnitude shorter penetration depth of fast electrons as compared to a metal target, due to strong electrical inhibition. This explains the enhanced electron flux observed at the rear of metalfilled PAA.

\section{CONCLUSION}

In conclusion, we have shown that a target with an array of parallel, $60 \mu \mathrm{m}$ long, nanochannels yields 60 -fold enhancement in hot electron flux, compared to a polished (unstructured) target. This is explained in terms of increased penetration of light pulse through an array of subwavelength nanoholes. We further observe that on launching an identical source of fast electrons into an alumina template in which nanochannels are partly filled with metallic $\mathrm{Cu}$, there is a four-fold increase in the hot electron flux as compared to a hollow alumina template, measured at the rear side of the targets. The enhancement in electron flux is attributed to large background electron conductivity provided by metallic doping of the nanochannels, favoring the setting up of return current. This innovative hybrid target design, incorporating two crucial features of fast electron physics, namely, efficient generation of hot electrons and their subsequent uninhibited transport, facilitates the realization of bright sources for charge particle accelerators, $\mathrm{x}$-ray backlighting, and proton radiography.

\section{ACKNOWLEDGMENTS}

G. R.K. acknowledges a J.C. Bose Fellowship grant from the Department of Science and Technology, Government of India.

[1] R.P. Drake, High-Energy-Density Physics (SpringerVerlag, Berlin Heidelberg, 2006).

[2] M. Tabak, J. Hammer, M.E. Glinsky, W. L. Kruer, S.C. Wilks, J. Woodworth, E. M. Campbell, M.D. Perry, and R. J. Mason, Phys. Plasmas 1, 1626 (1994).

[3] R. R. Freeman, D. Batani, S. Baton, M. Key, and R. Stephens, Fusion Sci. Technol. 49, 297 (2006) [www.ans.org/pubs/journals/fst/a_1150].

[4] V. Malka, J. Faure, Y. A. Gauduel, E. Lefebvre, A. Rousse, and K. T. Phuoc, Nat. Phys. 4, 447 (2008).

[5] M. M. Murnane, H.C. Kapteyn, M.D. Rosen, and R.W. Falcone, Science 251, 531 (1991).

[6] W. L. Kruer and P. Gibbon, The Physics of Laser Plasma Interactions (Addison-Wesley, New York, 1988).

[7] P. Gibbon, Short Pulse Laser Interactions with Matter: An Introduction (Imperial College Press, London, 2005).

[8] C.S. Liu and V.K. Tripathi, Interaction of Electromagnetic Waves with Electron Beams and Plasmas (World Scientific, Singapore, 1994).

[9] S. D. Baton et al., Phys. Rev. Lett. 91, 105001 (2003).

[10] H. Habara, K. Ohta, K. A. Tanaka, G. R. Kumar, M. Krishnamurthy, S. Kahaly, S. Mondal, M. K. Bhuyan, R. Rajeev, and J. Zheng, Phys. Rev. Lett. 104, 055001 (2010). 
[11] S. Kahaly, S. Yadav, W. Wang, S. Sengupta, Z. Sheng, A. Das, P. K. Kaw, and G. R. Kumar, Phys. Rev. Lett. 101, 145001 (2008).

[12] G. Hu et al., Phys. Plasmas 17, 033109 (2010)

[13] P. P. Rajeev, P. Taneja, P. Ayyub, A. S. Sandhu, and G. P. Kumar, Phys. Rev. Lett. 90, 115002 (2003).

[14] S. Mondal et al., Phys. Rev. B 83, 035408 (2011).

[15] Z. Zhao et al., Phys. Plasmas 17, 123108 (2010).

[16] S. Bagchi, P. Prem Kiran, K. Yang, A. M. Rao, M. K. Bhuyan, M. Krishnamurthy, and G. Ravindra Kumar, Phys. Plasmas 18, 014502 (2011).

[17] L. Cao, Y. Gu, Z. Zhao, L. Cao, W. Huang, W. Zhou, X. T. He, W. Yu, and M. Y. Yu Phys. Plasmas 17, 043103 (2010).

[18] T. Nishikawa, H. Nakano, N. Uesugi, M. Nakao, and H. Masuda, Appl. Phys. Lett. 75, 4079 (1999).

[19] H. Alfven, Phys. Rev. 55, 425 (1939).
[20] D. Batani et al., Phys. Rev. E 65, 066409 (2002).

[21] D. Bond, J. Hares, and J. Kilkenny, Phys. Rev. Lett. 45, 252 (1980).

[22] K. A. Tanaka, T. Yabuuchi, T. Sato, R. Kodama, Y. Kitagawa, T. Takahashi, T. Ikeda, Y. Honda, and S. Okuda, Rev. Sci. Instrum. 76, 013507 (2005).

[23] T. Yabuuchi et al., Phys. Plasmas 14, 040706 (2007).

[24] P. P. Rajeev, P. Ayyub, S. Bagchi, and G. R. Kumar, Opt. Lett. 29, 2662 (2004).

[25] H. Raether, Surface Plasmons on Smooth and Rough Surfaces and on Gratings (Springer-Verlag, Berlin Heidelberg, 1988).

[26] A. R Bell, J. R Davies, S. Guerin, and H. Ruhl, Plasma Phys. Controlled Fusion 39, 653 (1997).

[27] V. T. Tikhonchuk Phys. Plasmas 9, 1416 (2002).

[28] A. S. Sandhu, G. R. Kumar, S. Sengupta, A. Das, and P. K. Kaw, Phys. Rev. E 73, 036409 (2006). 